\title{
Dimensionamento da força de trabalho necessária às Unidades Hospitalares do Instituto Nacional de Câncer/MS
}

\author{
Virginia Maria Leite de Almeida' ${ }^{1}$ Alina Junqueira² ${ }^{2}$ Luiz Augusto Maltoni ${ }^{3}$ Luiz Cláudio Bruno ${ }^{4}$
}

\section{Resumo}

O objetivo deste estudo é contribuir para o estabelecimento de parâmetros adequados ao dimensionamento da força de trabalho, necessária ao atendimento de ações realizadas por unidades hospitalares especializadas de alta complexidade de assistência ao câncer. O estudo tem como campo de análise os quatro hospitais de atenção oncológica (HCI, HCII, HCIII e HCIV) e o Centro de Transplante de Medula Óssea do Instituto Nacional de Câncer. Suas principais atividades são diagnóstico, tratamento, reabilitação e cuidados paliativos de pacientes de câncer. A metodologia adotada relaciona a capacidade instalada, o tempo de execução das atividades, carga horária e o quantitativo das categorias profissionais necessárias ao adequado atendimento das demandas. O estudo aponta como estratégico o correto dimensionamento das equipes de enfermagem, para se obter resultados de produtividade e qualidade, considerando as características de continuidade ininterrupta e diversidade das atividades desenvolvidas pelas categorias de enfermagem, além do seu expressivo peso no conjunto da força de trabalho da área de saúde. Como o resultado do setor saúde está diretamente vinculado ao desempenho e dedicação dos profissionais nele envolvidos, o levantamento de necessidades de pessoal dos hospitais irá subsidiar o planejamento, o desenvolvimento e a gestão dessa força de trabalho.

Palavras-chave: Força de trabalho; Unidades hospitalares; Atenção oncológica

${ }^{1}$ Coordenadora de Recursos Humanos - CRH/INCA.

${ }^{2}$ Gerente de Divisão de Projetos Estratégicos - CRH/INCA.

${ }^{3}$ Coordenador de Assistência - INCA.

${ }^{4}$ Gerente de Divisão de Apoio Técnico - HCII/INCA. 


\section{INTRODUÇÃO}

O presente estudo tem como objetivo contribuir para o aprimoramento do conhecimento no campo do trabalho na área da saúde, através do estabelecimento de parâmetros adequados ao dimensionamento da força de trabalho necessária ao atendimento de ações, atividades e procedimentos realizados em unidades hospitalares especializadas de alta complexidade de assistência ao câncer.

Há algumas décadas, equivocadamente, o parâmetro utilizado para estimar a força de trabalho necessária a unidades hospitalares era o número de leitos. A partir da década de 1970, por "determinação" do desenvolvimento tecnológico, um novo olhar se volta para as atividades hospitalares quando um grande número dessas atividades passou a ser realizada fora do leito, ou seja, na esfera ambulatorial. Da mesma forma, a expansão das áreas de diagnóstico complementar, a utilização de novos equipamentos e procedimentos passaram a requerer um maior número de profissionais qualificados. Essa situação passou a exigir um novo parâmetro para estimar a força de trabalho que, a partir daí, não poderia mais ser determinada pelo número de leitos hospitalares.

Novos estudos foram desenvolvidos desde então, e entre eles está a revisão da literatura pertinente, realizada pelo Departamento de Saúde da Universidade de Leeds, Inglaterra, em 2003, que aponta cinco sistemas de planejamento da força de trabalho como os mais utilizados e adequados, atualmente.

Estudos voltados para o planejamento e gestão de recursos humanos em unidades hospitalares têm impacto direto não somente na produção de serviços, mas na qualidade do atendimento e determinam, ainda, açōes e atividades para a gestão do trabalho e para o desenvolvimento de recursos humanos.

O levantamento apontado neste estudo foi desenvolvido nas cinco unidades hospitalares do Instituto Nacional de Câncer (INCA) do Ministério da Saúde (MS).

Como produto, apresenta a distribuição dos recursos humanos necessários aos hospitais do INCA obtida através da utilização de uma metodologia que estabelece novos parâmetros para o planejamento e gestão da força de trabalho nas unidades hospitalares na área oncológica.

Os objetivos deste trabalho foram implementar uma metodologia para a definição de alocação de pessoal em unidades hospitalares especializadas e de alta complexidade e dimensionar o quadro de recursos humanos necessários ao desenvolvimento de atividades de assistência e de ensino nos cinco hospitais do Instituto Nacional de Câncer (INCA).

\section{INSTITUTO NACIONAL DE CÂNCER}

O INCA é o órgão do Ministério da Saúde responsável pelo controle do câncer no Brasil, através do desenvolvimento de ações nacionais visando à prevenção e ao controle do câncer.

Como integrante do Sistema de Ciência e Tecnologia (C\&T) desde 1993, exerce sua função pela integração de açôes assistenciais, educacionais e de investigação científico-tecnológica voltadas para o agravo do câncer.

A missão da Instituição é operacionalizada por objetivos estratégicos definidos de forma a permitir a integração de suas diversas áreas de atuação. São estes: - Promover a geração e a disseminação do conhecimento em atenção oncológica, na prevenção e no controle do câncer;

- Promover a descentralização das ações de atenção, prevenção e controle de câncer com abordagem multidisciplinar;

- Contribuir para o desenvolvimento tecnológico do complexo produtivo da saúde;

- Promover o desenvolvimento e a incorporação de uma cultura organizacional de gestão participativa e compartilhada, focada em resultados;

- Desenvolver e implantar ações internas e externas visando à melhor relação entre recursos alocados e resultados alcançados;

- Pactuar objetivos e metas institucionais para a avaliação dos desempenhos interno e externo;

- Ampliar as fontes de recursos financeiros que permitam o fortalecimento das ações nacionais em atenção, prevenção e controle do câncer.

\section{Estrutura}

A estrutura organizacional do INCA (Figura 1) inclui além de suas áreas técnicas, instâncias colegiadas de gestão.

O INCA é responsável pela prestação de serviços de assistência médico-hospitalar de referência, no âmbito do SUS, através dos quatro hospitais HCI, HCII, HCIII e HCIV e do Centro de Transplante de Medula Óssea (CEMO).

Como instituição integrante do Sistema de Ciência e Tecnologia, cabe, também, a essas unidades hospitalares, a formação de recursos humanos e a difusão de conhecimento, com participação ativa nos programas de ensino oferecidos pela Instituição, como: programas de residência, cursos de especialização, aperfeiçoamento e atualização, tanto para o nível técnico como para o superior, nas áreas: médica, enfermagem e nas grandes áreas da saúde. 


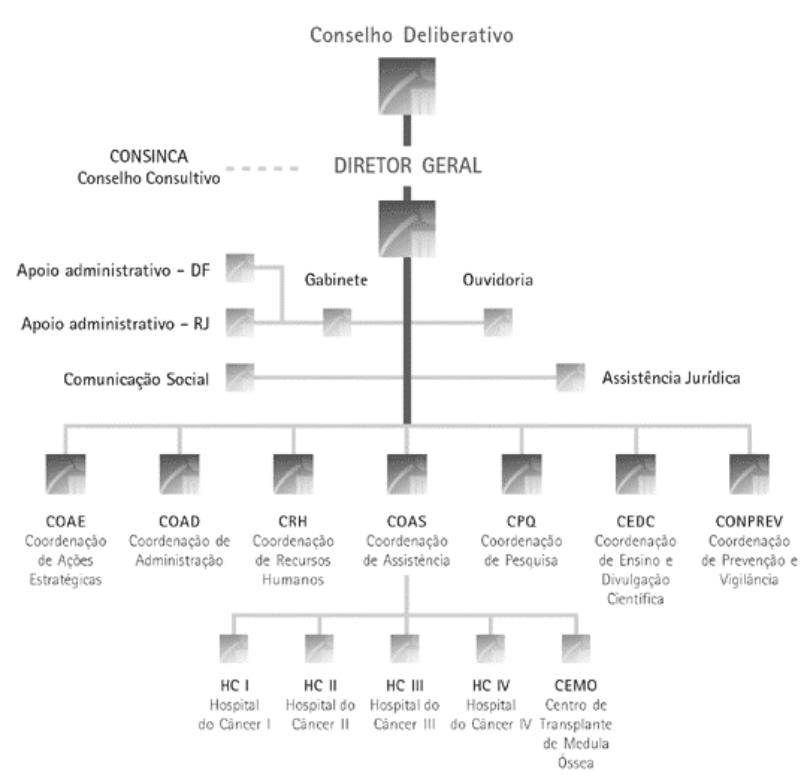

Figura 1. Estrutura organizacional do INCA Área Assistencial

Hospital do Câncer I (HC I): O Hospital do Câncer I (HC I) é a maior unidade hospitalar do INCA e a de maior complexidade. Atende a pacientes de todas as modalidades de câncer, inclusive pediátrico. O HC I possui além das Clínicas Médicas, incluindo os serviços de Dermatologia, Tórax, Urologia, Hematologia, a área de Oncologia Pediátrica e Oncologia Clínica, Psiquiatria e a Anestesiologia. A área cirúrgica contempla as clínicas de Cirurgia Plástica e Reparadora, Cirurgia de Cabeça e Pescoço, Cirurgia Abdominopélvica, Cirurgia Oncológica Pediátrica, Neurocirurgia e unidades de tratamento intensivo para adultos e crianças. A área complementar de diagnóstico e terapêutica compreende os Serviços de Radiologia, Física Médica, Radioterapia, Endoscopia, Patologia Clínica, Hemoterapia, Tecido Ósseo e Conectivo e Medicina Nuclear. No HCI, estão incluídas, também, as atividades da Divisão de Patologia que presta serviços de citopatologia e histopatologia para as Secretarias de 28 municípios no Estado do Rio de Janeiro. Compreende, ainda, como áreas de apoio: Psicologia, Fisioterapia, Fonoaudiologia, Odontologia, Serviço Social, Nutrição, Farmácia e Comissão de Infecção Hospitalar. A equipe de enfermagem encontrase presente em todas as áreas. O HC I possui 216 leitos e dez salas de cirurgia.

Hospital do Câncer II (HC II): O Hospital do Câncer II (HC II) é especializado em Ginecologia e Cancerologia Clínica. Recebe paciente adulto para tratamentos ambulatoriais e cirúrgicos de ginecologia, cancerologia clínica e internação. O HC II possui dois ambulatórios de Clínica Médica e três de Cancerologia Clínica. $\mathrm{Na}$ Ginecologia Clínica são sete ambulatórios e três Centros Cirúrgicos, além de Anestesia e um Centro de Terapia Intensiva. $\mathrm{Na}$ área complementar de diagnóstico e terapêutica, incluem-se os serviços de Radiologia, Hemoterapia e Endoscopia, e um Laboratório. O HC II compreende, ainda, como áreas de apoio, as atividades de Psicologia, Serviço Social, Fisioterapia, Nutrição, Farmácia e a Comissão de Infecção Hospitalar. Da mesma forma que nas demais unidades, a equipe de enfermagem encontra-se presente em todas as áreas. O HC II possui 83 leitos e três salas de cirurgia.

Hospital do Câncer III (HC III): O Hospital de Câncer III (HC III) é a unidade dedicada ao diagnóstico e tratamento do câncer de mama. Além dos serviços de Clínica Médica, Anestesia, Cancerologia Clínica e Mastologia, o HC II possui áreas complementares de diagnóstico e terapêuticas, a saber: Laboratório, Radiologia, Hemoterapia, Endoscopia e uma Unidade Transfusional. Como atividades de apoio, compreende Psicologia, Serviço Social, Fisioterapia, Nutrição, Farmácia e Comissão de Infecção Hospitalar. A Enfermagem, como nas outras unidades, foi tratada como integrante do conjunto das áreas do Hospital. O HC III possui 52 leitos e quatro salas de cirurgia.

Hospital de Câncer IV (HC IV): O Hospital de Câncer IV (HC IV) é a unidade especializada em cuidados paliativos com a finalidade de oferecer cuidados aos pacientes fora de possibilidades terapêuticas, através do atendimento domiciliar. Além da Divisão Médica, o HC IV compreende também os serviços de Fisioterapia, Nutrição, Serviço Social, Psicologia e Farmácia, que também atendem ao HC III. O HC IV possui 56 leitos e realiza, em média, 8.500 visitas domiciliares/ano.

Centro de Transplante de Medula Óssea (CEMO): O CEMO é a unidade de atendimento a pacientes com doenças hematológicas malignas e afecções correlatas, indicadas para transplante de medula óssea. A unidade integra o Sistema Nacional de Transplante, do Ministério da Saúde, coordenando o Banco de Células de Sangue de Cordão, Banco de Cordão Umbilical e Placentário (BSCUP), Divisão de Imunogenética e Transplante de Medula (DITRAN), Registro Nacional de Doadores de Medula Óssea (REDOME). O CEMO compreende uma Divisão Médica e ambulatórios de Psicologia, Fisioterapia, Serviço Social e Nutrição e, como área complementar de diagnóstico e terapêutica possui um Laboratório. O CEMO possui 12 leitos. 


\section{Produção anual do INCA}

A produção anual do INCA é apresentada na Tabela 1.

Tabela 1. Produção média nos Hospitais (julho de 2005)

\begin{tabular}{l|c}
\multicolumn{1}{c|}{ Procedimentos } & $\mathrm{n}$ \\
\hline Internações & 1.094 \\
Cirurgias & 976 \\
Quimioterapia - Aplicações + & 6.237 \\
Hormonioterapia & 12.087 \\
Radioterapia (campos) & 19.169 \\
Consultas médicas & 7 \\
Transplantes de medula óssea & 26.419 \\
Exames citopatológicos & 3.811 \\
Outras consultas &
\end{tabular}

\section{Força de trabalho do INCA}

A força de trabalho do INCA agrega servidores oriundos de diversas origens funcionais. Até a década de 1980, as necessidades de pessoal eram supridas através de concursos públicos, promovidos pelo antigo DASP. A ampliação de suas atividades determinou a necessidade de crescimento do seu quadro funcional, tanto qualitativa quanto quantitativamente, o que foi naquele momento viabilizado através de contratações pela Campanha Nacional de Combate ao Câncer (CNCC).

Em 1991, com a extinção da CNCC, o INCA perdeu o instrumento de reposição de seu quadro funcional e, tendo em vista o grande número de servidores aposentados após o advento da Lei 8112/90, falecimentos e afastamento legais, o déficit de pessoal agravou-se de forma alarmante. Ainda em 1991, buscando uma solução para o problema, foi constituída a Fundação Ary Frauzino (FAF), de apoio às atividades do INCA, de direito privado, dotada de autonomia patrimonial, administrativa e financeira.

Em 1992, foram incorporadas ao INCA duas unidades assistenciais: o Centro de Ginecologia Luiza Gomes de Lemos, oriundo da extinção das Pioneiras Sociais, e o Hospital de Oncologia, do extinto INAMPS.
As duas novas unidades tornaram mais complexa a situação funcional da Instituição por estarem também com grave déficit em seus quadros de pessoal.

Em 1993, por meio da Lei 8691/93, o INCA passou a integrar o Plano de Carreiras de Ciência e Tecnologia, sendo os seus profissionais enquadrados nos cargos de nível superior: de Pesquisador, Tecnologista e Analista em C\&T; de nível médio: Técnico e Assistente em C\&T; e de nível auxiliar: de Auxiliar e Auxiliar Técnico.

A partir de 1997, para que o INCA não se tornasse inviável, principalmente na assistência à população, intensificou-se o processo de reposição de pessoal através da FAF. O quadro funcional do INCA, em junho de 2005, estava constituído por 3.246 profissionais. Este quantitativo corresponde a 1.831 servidores contratados pelo MS (56,41\%) e 1.415 funcionários contratados pela FAF (43,59\%). Deste quantitativo, 1.986 (61\%) profissionais estão lotados na área assistencial.

A Tabela 2 apresenta a distribuição dessa força de trabalho pelas Unidades Assistenciais:

Embora seja na área assistencial que exista a maior concentração dos profissionais, é nesta área que se tem o maior problema, devido ao aumento da demanda em função do crescimento populacional e a grande concentração no INCA de, praticamente, todo o atendimento público assistencial na área oncológica.

Cabe ressaltar que o estrangulamento na área assistencial se reflete em todas as outras áreas da Instituição, principalmente nas áreas de Ensino, Pesquisa e Prevenção.

A dependência das Unidades Hospitalares em relação à força de trabalho necessária ao desenvolvimento de suas atividades vem aumentando ao longo do tempo em decorrência, principalmente, do aumento da complexidade dos procedimentos e do desenvolvimento tecnológico.

A definição de parâmetros para a quantificação e a distribuição da força de trabalho em Unidades Assistenciais possibilita a implementação de processos efetivos de planejamento e gestão, e a conseqüente melhoria da qualidade da assistência prestada à população.

Tabela 2. Distribuição da Força de Trabalho por Unidade Assistencial (junho de 2005)

\begin{tabular}{l|c|c}
\multicolumn{1}{c|}{ Unidades Hospitalares } & $\mathrm{n}$ & \% em relação ao total do INCA \\
\hline $\mathrm{HC} \mathrm{I}$ & 1.212 & $37 \%$ \\
$\mathrm{HC} \mathrm{II}$ & 323 & $10 \%$ \\
$\mathrm{HC} \mathrm{III}$ & 218 & $7 \%$ \\
$\mathrm{HC} \mathrm{IV}$ & 126 & $4 \%$ \\
$\mathrm{CEMO}$ & 107 & $3 \%$ \\
Total / Unidades Hospitalares & 1.986 & $61 \%$ \\
Total / INCA & 3.246 & $100 \%$ \\
\hline
\end{tabular}




\section{METODOLOGIA}

Em função da realidade do INCA, da necessidade de otimizar sua capacidade instalada e de potencializar seus recursos humanos, e com base nos estudos disponíveis relativos ao planejamento de força de trabalho, a metodologia adotada nesse estudo utilizou uma adaptação de dois métodos já experimentados, descritos pelo Dr. Kleith Hurst em Selecting and Applying Methods for Estimating the Size and Mix of Nursing Teams: a percepção de especialistas e de profissionais e o de tempo de execução de tarefas, e a introdução de um novo parâmetro, a capacidade instalada.

Dessa forma, este estudo considerou, como parâmetros para o dimensionamento da força de trabalho, a capacidade instalada das Unidades Assistenciais, a carga horária necessária à realização de cada atividade desenvolvida pelas áreas analisadas e a percepção de especialistas e de profissionais.

Como capacidade instalada dos Hospitais - HCI, HCII, HCIII, HCIV e CEMO - foram considerados: salas de ambulatório, de cirurgia, número de leitos, número de visitas domiciliares (para o HCIV), número de poltronas de atendimento, equipamentos e a demanda existente. Também foram abordadas as áreas em expansão, em vias de serem inauguradas, como a UPO do HC I e o $8^{\circ}$ andar do mesmo hospital que já está pronto, aguardando a contratação de recursos humanos; o ambulatório, o hospital-dia e a área pediátrica do CEMO, além do CTI do HC III.

A carga horária necessária à realização das atividades foi identificada através de entrevistas com os especialistas e chefias das áreas analisadas, correlacionando o tempo de execução das atividades com a capacidade instalada das unidades hospitalares, em consonância com as premissas do método de percepção de profissionais. A adaptação desse método teve como vantagens a facilidade de operacionalização e a agilidade de ajustes.

O dimensionamento da força de trabalho foi realizado, no período de maio a julho de 2005 , com cada chefe de Seção do HC I, HC II, HC III, HC IV e com o Diretor do CEMO, levando-se em conta, além da capacidade instalada, a atividade desenvolvida pela área, correlacionada com a categoria profissional necessária.

Para a exemplificação da metodologia adotada, são apresentadas, nas Tabelas 3 e 4, as etapas cumpridas, utilizando a situação do Centro de Transplante de Medula Óssea (CEMO).

Para cada atividade levantada, foram identificados o

Tabela 3. Capacidade Instalada e descrição de Atividades - Centro de Transplante de Medula Óssea - Categoria Médica

\begin{tabular}{|c|c|c|c|c|}
\hline \multirow{2}{*}{ Seção } & \multirow{2}{*}{ Atividade } & \multicolumn{3}{|c|}{ Capacidade Instalada } \\
\hline & & Equipamento & Sala & Leito \\
\hline Divisão Médica & Consultas & --- & 5 & --- \\
\hline Divisão Médica & Rotina do Hospital/Dia & --- & --- & 10 \\
\hline Divisão Médica & Biopsia & --- & 1 & --- \\
\hline Divisão Médica & Aspiração de Medula & --- & 1 & --- \\
\hline Divisão Médica & Colocação de Cateter Venoso & --- & 1 & --- \\
\hline Divisão Médica & Colocação de Cateter Longa Permanência & --- & 1 & --- \\
\hline Divisão Médica & Chefia & --- & --- & --- \\
\hline TOTAL & & --- & 10 & 10 \\
\hline
\end{tabular}

Tabela 4. Carga Horária, adicionais de férias e ensino e quantidade de pessoal - Centro de Transplante de Medula Óssea - Categoria Médica

\begin{tabular}{|c|c|c|c|c|c|c|c|c|c|c|c|}
\hline \multirow[t]{2}{*}{ Atividade } & \multicolumn{3}{|c|}{ Capacidade Instalada } & \multirow[t]{2}{*}{ Quantidade } & \multirow{2}{*}{$\begin{array}{c}\text { Carga } \\
\text { horária } \\
\text { diária }\end{array}$} & \multirow[t]{2}{*}{ Dias } & \multirow[t]{2}{*}{ Turno } & \multirow{2}{*}{$\begin{array}{l}\text { Carga } \\
\text { horária } \\
\text { semanal }\end{array}$} & \multirow{2}{*}{$\begin{array}{c}\text { Adicional } \\
\text { de férias } \\
(10 \%)\end{array}$} & \multirow{2}{*}{$\begin{array}{c}\text { Adicional } \\
\text { de ensino } \\
(10 \%)\end{array}$} & \multirow{2}{*}{$\begin{array}{l}\text { Quantidade de } \\
\text { Pessoal (40h) }\end{array}$} \\
\hline & Equipamento & Sala & Leito & & & & & & & & \\
\hline Consultas & --- & 5 & --. & 5 & 8 & 5 & -.- & 200 & 220 & 242 & 6 \\
\hline
\end{tabular}


número de profissionais por categoria, carga horária diária, tipos de turnos e plantões necessários ao funcionamento do serviço. Obtendo-se, dessa forma, a carga horária semanal.

Incidiu sobre esse resultado, um percentual de $10 \%$ a título de índice de segurança para cobrir férias, ausências e afastamentos. A área de enfermagem foi tratada de forma diferenciada de modo a atender à Resolução COFEN, no 293/2004 que determina que "o quantitativo de profissionais estabelecido deverá ser acrescido de um índice de segurança técnica (IST) não inferior a $15 \%$ do total".

Na seqüência, um acréscimo de $10 \%$ incidiu na carga horária semanal das categorias de nível superior destinadas à produção e difusão de conhecimento. Quando pertinente, foi sinalizada uma carga horária de $40 \mathrm{~h}$ semanais para a chefia.

Por fim, a carga horária foi dividida por $40 \mathrm{~h}$ semanais, em razão de o INCA estar integrado ao Plano de Carreiras para a área de Ciência e Tecnologia, que determina essa jornada de trabalho semanal, para se obter o quantitativo de pessoal necessário por atividade descrita, exceto para os cargos de Técnico em RX e Técnico em Radioterapia, cuja divisão foi por 24 horas, para atender à legislação específica.

O levantamento teve como foco as categorias de nível superior: médico, enfermeiro, fonoaudiólogo, farmacêutico, físico, fisioterapeuta, nutricionista, biólogo, psicólogo, assistente social, odontólogo, terapeuta ocupacional e as de nível técnico: técnico de enfermagem, técnico de farmácia, técnico de laboratório, técnico de radiologia, técnico de radioterapia e técnico de registro médico.

Para efeito deste trabalho, os profissionais que atuam na área de laboratório foram agrupados em níveis superior e técnico, sendo denominados de Analista de Laboratório as seguintes categorias: Biólogo, Biomédico, Bioquímico; enquanto os de nível técnico como Técnico de Laboratório: as categorias de Citotécnico, Técnico em Patologia e o Técnico em Laboratório. A área de gestão não foi incluída no levantamento.

$\mathrm{Na}$ Tabela 5, apresenta-se o resultado das etapas apresentadas.

\section{RESULTADOS}

A distribuição dos dados levantados nas cinco unidades hospitalares foi consolidada em termos de capacidade instalada, atividade desenvolvida, tempo de execução e categoria funcional.

A coleta e tabulação dos dados na área complementar de diagnóstico e terapêutica foram os mais difíceis tendo em vista a diversidade de atividades.

Quanto à equipe de enfermagem, os dados revelaram que o quantitativo necessário ao desenvolvimento do conjunto de atividades das cinco unidades estudadas representa $50 \%$ dos recursos humanos. Essa área também é bastante atuante nos programas de educação continuada e permanente da instituição. A área de enfermagem, certamente, é uma das áreas em que o dimensionamento de pessoal é estratégico para que se

Tabela 5. Quantitativo de pessoal necessário para a Divisão Médica - CEMO - Categoria Médica

\begin{tabular}{|c|c|c|c|c|c|c|c|c|c|c|c|c|}
\hline \multirow{2}{*}{ Atividade } & \multicolumn{3}{|c|}{ Capacidade Instalada } & \multirow{2}{*}{ Cargo } & \multirow{2}{*}{ Quantidade } & \multirow{2}{*}{$\begin{array}{c}\text { Carga } \\
\text { horária } \\
\text { diária }\end{array}$} & \multirow{2}{*}{ Dias } & \multirow{2}{*}{ Turno } & \multirow{2}{*}{$\begin{array}{c}\text { Carga } \\
\text { horária } \\
\text { semanal }\end{array}$} & \multirow{2}{*}{$\begin{array}{c}\text { Adicional } \\
\text { de férias } \\
(10 \%)\end{array}$} & \multirow{2}{*}{$\begin{array}{c}\text { Adicional } \\
\text { de ensino } \\
(10 \%)\end{array}$} & \multirow{2}{*}{$\begin{array}{c}\text { Quantidade } \\
\text { de pessoal } \\
\text { (/40h) }\end{array}$} \\
\hline & Equipamento & Sala & Leito & & & & & & & & & \\
\hline Consultas & & 5 & & Médico & 5 & 8 & 5 & & 200 & 220 & 242 & 6 \\
\hline $\begin{array}{l}\text { Rotina do } \\
\text { Hospital/Dia }\end{array}$ & & & 10 & Médico & 1 & 8 & 5 & & 40 & 44 & 48,4 & 1 \\
\hline Biopsia & & 1 & & Médico & 1 & 8 & 5 & & 40 & 44 & 48,4 & 1 \\
\hline $\begin{array}{l}\text { Aspiração de } \\
\text { medula }\end{array}$ & & 1 & & Médico & 2 & 8 & 1 & & 16 & 17,6 & 19,36 & 0 \\
\hline $\begin{array}{l}\text { Colocação de } \\
\text { cateter venoso }\end{array}$ & & 1 & & Médico & 1 & 4 & 1 & & 4 & 4,4 & 4,84 & 0 \\
\hline $\begin{array}{l}\text { Colocação de } \\
\text { cateter longa } \\
\text { permanência }\end{array}$ & & 1 & & Médico & 1 & 4 & 2 & & 8 & 8,8 & 9,68 & 0 \\
\hline Chefia & & & & Médico & 1 & 8 & 5 & & 40 & 40 & 40 & 1 \\
\hline
\end{tabular}


obtenham resultados de produtividade e de qualidade.

$\mathrm{Na}$ área médica, a proporção de pessoal em relação ao total correspondeu a $20 \%$.

Nas áreas de apoio, o percentual correspondente à necessidade de recursos humanos foi de $11 \%$. No HC IV, no entanto, em função das atividades de visitas domiciliares, essas atividades têm um impacto de $26 \%$ em relação ao total da unidade.

A Tabela 6 apresenta o consolidado necessário por categoria profissional para cada uma das Unidades Hospitalares.

A grande demanda (10\%) por profissionais da área de laboratório ocorre em função dos exames especializados e do grande quantitativo de análises diárias de exames citológicos.

A Tabela 7 apresenta o quantitativo de recursos humanos existente, correlacionado com a força de trabalho necessária. $\mathrm{O}$ resultado apontou para um déficit total de 677 profissionais, distribuído pelo conjunto de categorias profissionais em todas as Unidades Hospitalares.

\section{CONCLUSÕES}

A sistematização e a divulgação deste trabalho visam a estabelecer bases para o desenvolvimento de outros estudos relacionados ao planejamento e gestão da força de trabalho em unidades hospitalares, contribuindo dessa forma para o estabelecimento de políticas voltadas para o processo de regulação e organização do trabalho.

Entende-se, portanto, o resultado deste trabalho como um instrumento importante tanto para a área de assistência e em especial para a área de Recursos Humanos e seus subsistemas de recrutamento, seleção e capacitação, com a ampliação da produção de conhecimento sobre a força de trabalho na área de assistência oncológica.

Os resultados apresentados podem servir como informação estratégica para o fortalecimento de encaminhamentos de resolução aos problemas das Unidades Hospitalares quanto ao déficit e conseqüente necessidade de complementação da força de trabalho necessária ao desenvolvimento eficiente e de qualidade das atividades das áreas.

Tabela 6. Quantitativo necessário por Unidade Hospitalar e por categoria profissional

\begin{tabular}{l|c|c|c|c|c|c}
\multicolumn{1}{c|}{ Cargo } & CEMO & HCI & HC II & HC III & HC IV & Total Global \\
\hline Assistente Social & 2 & 20 & 7 & 7 & 16 & 52 \\
Biólogo & 31 & 101 & & 9 & & 142 \\
Citotécnico & & 37 & & & & 37 \\
Enfermeiro & 58 & 274 & 96 & 78 & 38 & 545 \\
Farmacêutico & & 30 & 18 & 17 & & 65 \\
Físico & & 14 & & 4 & & 17 \\
Fisioterapeuta & 2 & 28 & 10 & 12 & 9 & 61 \\
Fonoaudiólogo & & 6 & & & & 6 \\
Médico & 34 & 297 & 79 & 76 & 35 & 521 \\
Nutricionista & 2 & 32 & 14 & 12 & 11 & 71 \\
Odontólogo & 1 & 5 & & & & 5 \\
Psicólogo & & 38 & 4 & 6 & 7 & 32 \\
Técnico de Farmácia & & 30 & 3 & 5 & & 63 \\
Técnico de Hemoterapia & 11 & 38 & 11 & 2 & & 38 \\
Técnico de Laboratório & & 47 & 21 & 13 & & 63 \\
Técnico de Raio X & 7 & & & & 82 \\
Técnico de Registro Médico & 25 & 434 & 193 & 102 & 36 & 790 \\
Técnico de Enfermagem & & 58 & & 7 & & 65 \\
Técnico de Radioterapia & & 2 & & & & 2 \\
Terapeuta ocupacional & 168 & 1.511 & 469 & 362 & 153 & 2.663 \\
Total & & & & & & \\
\hline
\end{tabular}

Referência: junho/2005

Obs: 1 . Inclui $10 \%$ de índice de segurança para todas as categorias e $15 \%$ para Enfermeiro e Técnico em enfermagem.

2. Inclui o percentual de $10 \%$ de ensino para as categorias de nível superior. 
Tabela 7. Comparativo entre o quadro de pessoal existente e o levantamento de necessidade por Unidade Hospitalar

\begin{tabular}{|c|c|c|c|c|c|c|c|c|c|c|c|c|c|c|c|c|c|c|}
\hline \multirow{2}{*}{$\begin{array}{c}\text { Função } \\
\text { Cargo Correlação }\end{array}$} & \multicolumn{3}{|c|}{ CEMO } & \multicolumn{3}{|c|}{ HC I } & \multicolumn{3}{|c|}{ HC II } & \multicolumn{3}{|c|}{ HC III } & \multicolumn{3}{|c|}{ HC IV } & \multicolumn{3}{|c|}{ TOTAL } \\
\hline & $\mathbf{Q P}$ & LP & SALDO & $Q P$ & LP & SALDO & $Q P$ & LP & SALDO & $Q P$ & LP & SALDO & $Q P$ & LP & SALDO & QP & LP & SALDO \\
\hline An de Laboratório & 19 & 31 & -13 & 38 & 101 & -63 & 2 & 0 & 2 & 8 & 9 & -1 & 1 & 0 & 1 & 67 & 142 & -74 \\
\hline Assistente Social & 1 & 2 & -1 & 15 & 20 & -5 & 9 & 7 & 2 & 3 & 7 & -4 & 9 & 16 & -7 & 36 & 52 & -16 \\
\hline Enfermeiro & 46 & 58 & -12 & 179 & 274 & -95 & 50 & 96 & -46 & 38 & 78 & -40 & 24 & 38 & -14 & 337 & 545 & -208 \\
\hline Farmacêutico & 0 & 0 & 0 & 13 & 30 & -17 & 7 & 18 & -11 & 3 & 17 & -14 & 1 & 0 & 1 & 23 & 65 & -41 \\
\hline Físico & 0 & 0 & 0 & 15 & 14 & 1 & 0 & 0 & 0 & 0 & 4 & -4 & 0 & 0 & 0 & 15 & 17 & -2 \\
\hline Fisioterapeuta & 0 & 2 & -2 & 11 & 28 & -18 & 3 & 10 & -7 & 4 & 12 & -7 & 1 & 9 & -8 & 19 & 61 & -42 \\
\hline Fonoaudiologo & 0 & 0 & 0 & 2 & 6 & -4 & 0 & 0 & 0 & 0 & 0 & 0 & 0 & 0 & 0 & 2 & 6 & -4 \\
\hline Médico & 18 & 34 & -15 & 268 & 297 & -29 & 65 & 79 & -14 & 51 & 76 & -24 & 23 & 35 & -13 & 425 & 521 & -96 \\
\hline Nutricionista & 0 & 2 & -2 & 11 & 32 & -21 & 2 & 14 & -12 & 2 & 12 & -10 & 1 & 11 & -10 & 16 & 71 & -56 \\
\hline Odontólogo & 0 & 0 & 0 & 9 & 5 & 4 & 0 & 0 & 0 & 0 & 0 & 0 & 0 & 0 & 0 & 9 & 5 & 4 \\
\hline Psicólogo & 1 & 1 & 0 & 5 & 14 & -9 & 1 & 4 & -3 & 3 & 6 & -3 & 1 & 7 & -6 & 11 & 32 & -21 \\
\hline Técnico de Enfermagem & 17 & 25 & -8 & 408 & 434 & -26 & 152 & 193 & -41 & 85 & 102 & -17 & 63 & 36 & 27 & 724 & 790 & -66 \\
\hline Técnico de Farmácia & 0 & 0 & 0 & 12 & 38 & -26 & 4 & 13 & -9 & 1 & 0 & 1 & 4 & 13 & -9 & 21 & 63 & -42 \\
\hline Técnico de Laboratório & 6 & 11 & -5 & 121 & 105 & 15 & 11 & 15 & -3 & 3 & 7 & -4 & 0 & 0 & 0 & 141 & 138 & 2 \\
\hline Técnico de Radiologia & 0 & 0 & 0 & 55 & 47 & 8 & 18 & 21 & -3 & 15 & 13 & 2 & 0 & 0 & 0 & 88 & 82 & 6 \\
\hline $\begin{array}{l}\text { Técnico de } \\
\text { Radioterapia }\end{array}$ & 0 & 0 & 0 & 49 & 58 & -9 & 0 & 0 & 0 & 2 & 7 & -5 & 0 & 0 & 0 & 51 & 65 & -14 \\
\hline $\begin{array}{l}\text { Técnico de Registro } \\
\text { Médico }\end{array}$ & 0 & 0 & & & 7 & -7 & & 0 & 0 & & 0 & 0 & & 0 & 0 & 0 & 7 & -7 \\
\hline Terapeuta Ocupacional & 0 & 0 & 0 & 2 & 2 & 0 & 0 & 0 & 0 & 0 & 0 & 0 & 0 & 0 & 0 & 2 & 2 & 0 \\
\hline Total & 107 & 168 & -61 & 1.212 & 1.511 & -299 & 323 & 469 & -146 & 218 & 350 & -132 & 126 & 165 & -39 & 1.986 & 2.663 & -677 \\
\hline
\end{tabular}

Referência: Junho/2005

$\mathrm{QP}=$ Quadro de Pessoal; LP=Levantamento de Pessoal

\section{BIBLIOGRAFÍA CONSULTADA}

1. Barría MS, Contardo N, Caravantes R, Monasterio H. Manual de Análisis de la Dotación de Personal en Establecimientos de Salud. OPAS (Organización Panamericana de Salud); 2004.

2. Bittar Olímpio JNV. Distribuição dos Recursos Humanos em Oito Hospitais Gerais de São Paulo. Rev Panam Salud Pública. 1977;2(1).

3. Brasil. Conselho Federal deEnfermagem (COFEN). Resolução COFEN no 293/2004 de 21 de setembro de 2004.

4. Brasil. Ministério da Saúde. Instituto Nacional do Câncer. Relatório Anual. Rio de Janeiro: INCA; 2003.
5. Brasil. Ministério da Saúde. Instituto Nacional do Câncer. Relatório Anual. Rio de Janeiro: INCA; 2004.

6. Brasil. Ministério da Saúde. Instituto Nacional do Câncer. Relatório Anual. Rio de Janeiro: INCA; 2005.

7. Hurst K. Selecting applying methods for estimating the size and mix of nursing teams. Nuffield Institute for Health. Leeds; 2002.

8. Shipp PJ. Workload Indicators of staffing need. WHO: Geneva;1998.

9. Zurn P, Poz MRD, Adams BS, Adams O. Imbalance in the health workforce. WHO: Human Resources for Health. Geneva; 2004. 